\title{
NONLINEAR, NONHOMOGENEOUS PARAMETRIC NEUMANN PROBLEMS
}

\author{
Sergiu Aizicovici - Nikolaos S. Papageorgiou — Vasile Staicu
}

\begin{abstract}
We consider a parametric nonlinear Neumann problem driven by a nonlinear nonhomogeneous differential operator, with a Carathéodory reaction $f$ which is $p$-superlinear in the second variable, but not necessarily satisfying the usual in such cases Ambrosetti-Rabinowitz condition. We prove a bifurcation type result describing the dependence of positive solutions on the parameter $\lambda>0$, show the existence of a smallest positive solution $\bar{u}_{\lambda}$ and investigate properties of the map $\lambda \mapsto \bar{u}_{\lambda}$. Finally, we show the existence of nodal solutions.
\end{abstract}

\section{Introduction}

In this paper we study the following nonlinear parametric Neumann problem:

$\left(\mathrm{P}_{\lambda}\right) \quad \begin{cases}-\operatorname{div} a(D u(z))+\lambda|u(z)|^{p-2} u(z)=f(z, u(z)) & \text { in } \Omega, \\ \frac{\partial u}{\partial n}=0 & \text { on } \partial \Omega, \lambda>0,\end{cases}$

2010 Mathematics Subject Classification. 35J66, 35J70, 35J92.

Key words and phrases. Positive solutions; nonlinear nonhomogeneous differential operator; nonlinear regularity; nonlinear maximum principle; bifurcation type result; nodal solutions.

The third author gratefully acknowledges the partial support by Portuguese funds through CIDMA - Center for Research and Development in Mathematics and Applications, and the Portuguese Foundation for Science and Technology (FCT), within the project PEstOE/MAT/UI4106/2014, and through the Sabbatical Fellowship SFRH/BSAB/113647/2015 during his sabbatical leave when he visited the Department of Information Engineering, Computer Science and Mathematics (DISIM) of the University of L'Aquila (Italy). The hospitality and partial support of DISIM are gratefully acknowledged. 
$1<p<\infty$. Here $\Omega \subset \mathbb{R}^{N}$ is a bounded domain with a $C^{2}$-boundary $\partial \Omega$. The map $a: \mathbb{R}^{N} \rightarrow \mathbb{R}^{N}$ is continuous, strictly monotone and satisfies certain regularity conditions which are listed in hypotheses H(a) (see Section 2). These hypotheses are general enough to incorporate in our framework many differential operators of interest, such as the $p$-Laplacian. Also $\lambda>0$ is a parameter and $f$ is a Carathéodory function (i.e. for all $x \in \mathbb{R}, z \mapsto f(z, x)$ is measurable and for almost all $z \in \Omega, x \mapsto f(z, x)$ is continuous) which exhibits a ( $p-1)$-superlinear growth in the second variable, but not necessarily satisfying the usual in such cases Ambrosetti-Rabinowitz condition (AR-condition for short).

Our work is motivated by a recent paper of Motreanu, Motreanu and Papageorgiou [18], who produced constant sign and nodal solutions. Our results complement and improve those of [18]. More precisely, the authors in [18] produced positive solutions for problem $\left(\mathrm{P}_{\lambda}\right)$ but did not give the precise dependence of the set of positive solutions on the parameter $\lambda>0$. Here, we prove a bifurcation-type theorem for large values of $\lambda$, which gives a complete picture of the set of positive solutions as the parameter varies. Moreover, in [18] nodal (that is, sign-changing) solutions were produced only for the particular case of equations driven by the $p$-Laplacian. In contrast, here we generate nodal solutions for the general case. We stress that the $p$-Laplacian differential operator is homogeneous, while the differential operator in $\left(\mathrm{P}_{\lambda}\right)$ is not. Hence, the methods and techniques used in [18] fail in the present setting, and so a new approach is needed. Finally, we mention that a bifurcation near infinity for a different class of $p$-Laplacian Dirichlet problems was recently produced by Gasinski and Papageorgiou [12].

In the next section, we review the main mathematical tools which will be used in this paper. We also present the hypotheses on the map $y \mapsto a(y)$ and state some useful consequences of them.

\section{Mathematical background}

Let $(X,\|\cdot\|)$ be a Banach space and $X^{*}$ be its topological dual. By $\langle\cdot, \cdot\rangle$ we denote the duality brackets for the pair $\left(X^{*}, X\right)$ and $\stackrel{\mathrm{w}}{\longrightarrow}$ will designate the weak convergence.

Let $\varphi \in C^{1}(X)$. We say that $x^{*} \in X$ is a critical point of $\varphi$ if $\varphi^{\prime}\left(x^{*}\right)=0$. If $x^{*} \in X$ is a critical point of of $\varphi$ then $c=\varphi\left(x^{*}\right)$ is a critical value of $\varphi$. We say that $\varphi$ satisfies the "Palais-Smale condition" (PS-condition for short), if the following holds:

"Every sequence $\left\{u_{n}\right\}_{n \geq 1} \subseteq X$ such that $\left\{\varphi\left(u_{n}\right)\right\}_{n \geq 1}$ is bounded in $\mathbb{R}$ and $\varphi^{\prime}\left(u_{n}\right) \rightarrow 0$ in $X^{*}$ as $n \rightarrow \infty$ admits a strongly convergent subsequence." 
This compactness-type condition on the functional $\varphi$ leads to a deformation theorem from which one can derive the minimax theory of critical values of $\varphi$. One of the main results in that theory is the so-called "mountain pass theorem", which we recall here.

TheOREM 2.1. If $\varphi \in C^{1}(X)$ satisfies the PS-condition, $u_{0}, u_{1} \in X$ with $\left\|u_{1}-u_{0}\right\|>\rho>0$ and

$$
\begin{aligned}
\max \left\{\varphi\left(u_{0}\right), \varphi\left(u_{1}\right)\right\} & <\inf \left\{\varphi(u):\left\|u-u_{0}\right\|=\rho\right\}=: m_{\rho}, \\
c: & : \inf _{\gamma \in \Gamma} \max _{t \in[0,1]} \varphi(\gamma(t)),
\end{aligned}
$$

where $\Gamma=\left\{\gamma \in C([0,1], X): \gamma(0)=u_{0}, \gamma(1)=u_{1}\right\}$, then $c \geq m_{\rho}$ and $c$ is a critical value of $\varphi$.

The main spaces that we will use in the analysis of problem $\left(\mathrm{P}_{\lambda}\right)$ are the Sobolev space $W^{1, p}(\Omega)$ and the Banach space $C^{1}(\bar{\Omega})$. The latter is an ordered Banach space with positive cone

$$
\mathcal{C}_{+}=\left\{u \in C^{1}(\bar{\Omega}): u(z) \geq 0 \text { for all } z \in \bar{\Omega}\right\} .
$$

This cone has a nonempty interior given by

$$
\operatorname{int} \mathcal{C}_{+}=\left\{u \in \mathcal{C}_{+}: u(z)>0 \text { for all } z \in \bar{\Omega}\right\} .
$$

By $\|\cdot\|$ we denote the norm of the Sobolev space $W^{1, p}(\Omega)$, that is

$$
\|u\|=\left[\|u\|_{p}^{p}+\|D u\|_{p}^{p}\right]^{1 / p} \text { for all } u \in W^{1, p}(\Omega),
$$

where $\|\cdot\|_{p}$ denotes the norm in $L^{p}(\Omega)\left(\right.$ or $\left.L^{p}\left(\Omega, \mathbb{R}^{N}\right)\right)$.

Also, by $\|\cdot\|$ we denote the $\mathbb{R}^{N}$-norm. However, no confusion is possible, since it will be clear from the context which norm is used. The inner product in $\mathbb{R}^{N}$ will be denoted by $(\cdot, \cdot)_{\mathbb{R}^{N}}$.

Let $\theta \in C^{1}(0, \infty)$ and assume that there exist constants $\widehat{C}, C_{0}, C_{1}, C_{2}>0$ such that

$$
\widehat{C} \leq \frac{t \theta^{\prime}(t)}{\theta(t)} \leq C_{0} \quad \text { and } \quad C_{1} t^{p-1} \leq \theta(t) \leq C_{2}\left(1+t^{p-1}\right) \quad \text { for all } t>0,
$$

with $1<p<\infty$. The hypotheses on the map $a: \mathbb{R}^{N} \rightarrow \mathbb{R}^{N}$ are the following:

$\mathrm{H}(\mathrm{a}) a(y)=a_{0}(\|y\|) y$ for all $y \in \mathbb{R}^{N}$ with $a_{0}(t)>0$ for all $t>0$ and

(i) $a_{0} \in C^{1}(0, \infty), t \mapsto t a_{0}(t)$ is strictly increasing in $(0, \infty), t a_{0}(t) \rightarrow$ $0^{+}$as $t \rightarrow 0^{+}$and

$$
\lim _{t \rightarrow 0^{+}} \frac{t a_{0}^{\prime}(t)}{a_{0}(t)}>-1 ;
$$

(ii) for some $C_{3}>0$ and all $y \in \mathbb{R}^{N} \backslash\{0\}$

$$
\|\nabla a(y)\| \leq C_{3} \frac{\theta(\|y\|)}{\|y\|} ;
$$


(iii) for all $y \in \mathbb{R}^{N} \backslash\{0\}$ and all $\xi \in \mathbb{R}^{N}$

$$
\frac{\theta(\|y\|)}{\|y\|}\|\xi\|^{2} \leq(\nabla a(y) \xi, \xi)_{\mathbb{R}^{N}}
$$

(iv) if $G_{0}(t)=\int_{0}^{t} s a_{0}(s) d s$, then there exists $\xi_{0}>0$ such that

$$
p G_{0}(t)-t^{2} a_{0}(t) \geq-\xi_{0} \quad \text { for all } t>0 ;
$$

(v) there exist $\tau, q \in(1, p)$ such that

$$
t \mapsto G_{0}\left(t^{1 / \tau}\right) \text { is convex and } \lim _{t \rightarrow 0^{+}} \frac{G_{0}(t)}{t^{q}}=0 .
$$

REMARK 2.2. The hypotheses H(a) (i)-(iii) were motivated by the regularity results of Lieberman [15] (p. 320) and the nonlinear maximum principle of Pucci and Serrin [25] (pp.111,120). Hypotheses H(a) (iv)-(v) are particular for our problem here, but they are quite general and are satisfied by many differential operators of interest (see Examples 2.5 below). Hypotheses $\mathrm{H}(\mathrm{a})$ imply that the primitive $G_{0}$ is strictly convex and strictly increasing.

We set

$$
G(y)=G_{0}(\|y\|) \quad \text { for all } y \in \mathbb{R}^{N} .
$$

Evidently $G$ is convex and differentiable on $\mathbb{R}^{N}$. We have

$$
\nabla G(y)=G_{0}^{\prime}(\|y\|) \frac{y}{\|y\|}=a_{0}(\|y\|) y \quad \text { for all } y \in \mathbb{R}^{N} \backslash\{0\}, \quad \nabla G(0)=0 .
$$

Since $G$ is convex and $G(0)=0$, we have

$$
G(y) \leq(a(y), y)_{\mathbb{R}^{N}} \quad \text { for all } y \in \mathbb{R}^{N} .
$$

The next lemma summarizes the main properties of $G$ and is an easy consequence of hypotheses $\mathrm{H}(\mathrm{a})$ (i)-(iii).

Lemma 2.3. If hypotheses $\mathrm{H}(\mathrm{a})(\mathrm{i})-(\mathrm{iii})$ hold, then:

(a) the map $y \mapsto a(y)$ is continuous and strictly monotone, hence maximal monotone too;

(b) $\|a(y)\| \leq C_{4}\left(1+\|y\|^{p-1}\right)$ for some $C_{4}>0$ and all $y \in \mathbb{R}^{N}$;

(c) $(a(y), y)_{\mathbb{R}^{N}} \geq C_{1}\|y\|^{p} /(p-1)$ for all $y \in \mathbb{R}^{N}$.

This lemma together with (2.1) and (2.2) leads to the following growth estimates for $G$.

Corollary 2.4. If hypotheses $\mathrm{H}(\mathrm{a})$ (i)-(iii) hold, then

$$
\frac{C_{1}}{p(p-1)}\|y\|^{p} \leq G(y) \leq C_{5}\left(1+\|y\|^{p}\right) \quad \text { for some } C_{5}>0 \text {, all } y \in \mathbb{R}^{N} \text {. }
$$

EXAMPLES 2.5. The following maps $a$ satisfy hypotheses H(a): 
(a) $a(y)=\|y\|^{p-2} y$ with $1<p<\infty$. This map corresponds to the $p$-Laplace differential operator defined by

$$
\triangle_{p} u=\operatorname{div}\left(\|D u\|^{p-2} D u\right) \text { for all } u \in W^{1, p}(\Omega) .
$$

(b) $a(y)=\|y\|^{p-2} y+\|y\|^{q-2} y$ with $1<q<p<\infty$. This map corresponds to the $(p, q)$-Laplacian defined by

$$
\triangle_{p} u+\triangle_{q} u \text { for all } u \in W^{1, p}(\Omega) .
$$

Such operators arise in many physical applications (see Cherfils and Ilyasov [6] and the references therein). Recently there have been existence and multiplicity results for equations driven by such operators. We mention the works of Aizicovici, Papageorgiou and Staicu [4], Cingolani and Degiovanni [7], Mugnai and Papageorgiou [22], Papageorgiou and Radulescu [23], Sun [26].

(c) $a(y)=\left(1+\|y\|^{2}\right)^{(p-2) / 2} y$ with $1<p<\infty$. This map corresponds to the generalized $p$-mean curvature differential operator defined by

$$
\operatorname{div}\left(\left(1+\|D u\|^{2}\right)^{(p-2) / 2} D u\right) \quad \text { for all } u \in W^{1, p}(\Omega) .
$$

(d) $a(y)=\|y\|^{p-2} y+\frac{\|y\|^{q-2} y}{1+\|y\|^{q}}$ with $1<q \leq p$.

(e) $a(y)=\left\{\begin{array}{ll}\|y\|^{p-1} y & \text { if }\|y\|<1 \\ 2\|y\|^{p-2} y-\|y\|^{p-3} y & \text { if } 1<\|y\|\end{array}\right.$ with $1<p<\infty$.

Let $A: W^{1, p}(\Omega) \rightarrow W^{1, p}(\Omega)^{*}$ be the nonlinear map defined by

$$
\langle A(u), y\rangle=\int_{\Omega}(a(D u), D y)_{\mathbb{R}^{N}} d z \quad \text { for all } u, y \in W^{1, p}(\Omega) .
$$

From Papageorgiou, Rocha and Staicu [24] we have:

Proposition 2.6. If hypotheses $\mathrm{H}(\mathrm{a})(\mathrm{i})$-(iii) hold, then the map $A: W^{1, p}(\Omega)$ $\rightarrow W^{1, p}(\Omega)^{*}$ defined by (2.3) is demicontinuous, strictly monotone (hence maximal monotone too) and of type $(S)_{+}$(that is, if $u_{n} \stackrel{\mathrm{w}}{\longrightarrow} u$ in $W^{1, p}(\Omega)$ and

$$
\limsup _{n \rightarrow \infty}\left\langle A\left(u_{n}\right), u_{n}-u\right\rangle \leq 0,
$$

then $u_{n} \rightarrow u$ in $W^{1, p}(\Omega)$ as $\left.n \rightarrow \infty\right)$.

Let $f_{0}: \Omega \times \mathbb{R} \rightarrow \mathbb{R}$ be a Carathéodory function such that

$$
\left|f_{0}(z, x)\right| \leq a_{0}(z)\left(1+|x|^{r-1}\right) \quad \text { for a.a. } z \in \Omega \text {, all } x \in \mathbb{R}
$$

with $a_{0} \in L^{\infty}(\Omega)_{+}$and $1<r<p^{*}$, where

$$
p^{*}:= \begin{cases}\frac{N p}{N-p} & \text { if } p<N \\ +\infty & \text { if } p \geq N .\end{cases}
$$


We set

$$
F_{0}(z, x)=\int_{0}^{x} f_{0}(z, s) d s
$$

and consider the $C^{1}$-functional $\varphi_{0}: W^{1, p}(\Omega) \rightarrow \mathbb{R}$ defined by

$$
\varphi_{0}(u)=\int_{\Omega} G(D u(z)) d z-\int_{\Omega} F_{0}(z, u(z)) d z \quad \text { for all } u \in W^{1, p}(\Omega) .
$$

From Motreanu and Papageorgiou [21], we have:

Proposition 2.7. If hypotheses $\mathrm{H}(\mathrm{a})$ (i)-(iii) hold, $\varphi_{0}: W^{1, p}(\Omega) \rightarrow \mathbb{R}$ is as defined above and $u_{0} \in W^{1, p}(\Omega)$ is a local $C^{1}(\bar{\Omega})$-minimizer of $\varphi_{0}$, that is, there exists $\rho_{0}>0$ such that

$$
\varphi_{0}\left(u_{0}\right) \leq \varphi_{0}\left(u_{0}+h\right) \quad \text { for all } h \in C^{1}(\bar{\Omega}) \text { with }\|h\|_{C^{1}(\bar{\Omega})} \leq \rho_{0},
$$

then $u_{0} \in C_{0}^{1, \alpha}(\bar{\Omega})$ for some $\alpha \in(0,1)$ and $u_{0}$ is also a $W^{1, p}(\Omega)$-minimizer of $\varphi_{0}$, that is, there exists $\rho_{1}>0$ such that

$$
\varphi_{0}\left(u_{0}\right) \leq \varphi_{0}\left(u_{0}+h\right) \quad \text { for all } h \in W^{1, p}(\Omega) \text { with }\|h\| \leq \rho_{1} .
$$

Let $X$ be a Banach space, $\varphi \in C^{1}(X)$ and $c \in \mathbb{R}$. We introduce the following sets:

$$
\begin{aligned}
\varphi^{c} & =\{u \in X: \varphi(u) \leq c\}, \\
K_{\varphi} & =\left\{u \in X: \varphi^{\prime}(u)=0\right\}, \\
K_{\varphi}^{c} & =\left\{u \in K_{\varphi}: \varphi(u)=c\right\} .
\end{aligned}
$$

For every topological pair $\left(Y_{1}, Y_{2}\right)$ with $Y_{2} \subset Y_{1} \subset X$ and every integer $k \geq 0$, by $H_{k}\left(Y_{1}, Y_{2}\right)$ we denote the $k^{\text {th }}$-relative singular homology group with integer coefficients.

Given an isolated $u \in K_{\varphi}^{c}$, the critical groups of $\varphi$ at $u$ are defined by

$$
C_{k}(\varphi, u)=H_{k}\left(\varphi^{c} \cap U,\left(\varphi^{c} \cap U\right) \backslash\{u\}\right), \quad \text { for all integers } k \geq 0,
$$

where $U$ is a neighbourhood of $u$ such that $K_{\varphi} \cap \varphi^{c} \cap U=\{u\}$. The excision property of the singular homology implies that the above definition is independent of the particular choice of the neighbourhood $U$.

Finally we outline some additional notations used in this paper. By $|\cdot|_{N}$ we denote the Lebesgue measure on $\mathbb{R}^{N}$. Given $x \in \mathbb{R}$, we define $x^{ \pm}=\max \{ \pm x, 0\}$.

For $u \in W^{1, p}(\Omega)$ we set $u^{ \pm}(\cdot)=u(\cdot)^{ \pm}$. We know that

$$
u^{ \pm} \in W^{1, p}(\Omega), \quad|u|=u^{+}+u^{-}, \quad u=u^{+}-u^{-} .
$$

Given a measurable function $h: \Omega \times \mathbb{R} \rightarrow \mathbb{R}$ (for example, a Carathéodory function), we define

$$
N_{h}(u)(\cdot)=h(\cdot, u(\cdot)) \quad \text { for all } u \in W^{1, p}(\Omega)
$$


(the Nemitskiŭ operator corresponding to $h$ ). Evidently $z \mapsto N_{h}(u)(z)=h(z, u(z))$ is measurable.

\section{Positive solutions}

In this section, we prove a bifurcation-type theorem describing the set of positive solutions of $\left(\mathrm{P}_{\lambda}\right)$ as $\lambda>0$ varies. We impose the following conditions on the reaction $f$ :

$\left(\mathrm{H}_{1}\right) f: \Omega \times \mathbb{R} \rightarrow \mathbb{R}$ is a Carathéodory function such that $f(z, 0)=0$ almost everywhere in $\Omega, f(z, x)>0$ for all $x>0$ and

(i) there exists $a \in L^{\infty}(\Omega)_{+}$such that $f(z, x) \leq a(z)\left(1+x^{r-1}\right)$ for almost all $z \in \Omega$, all $x \geq 0$, with $p<r<p^{*}$;

(ii) if $F(z, x)=\int_{0}^{x} f(z, s) d s$ then $\lim _{x \rightarrow+\infty} \frac{F(z, x)}{x^{p}}=+\infty \quad$ uniformly for a.a. $z \in \Omega$;

(iii) there exist $\mu \in\left(\max \{(r-p) N / p, 1\}, p^{*}\right)$ and $\beta_{0}>0$ such that $\beta_{0} \leq \liminf _{x \rightarrow+\infty} \frac{f(z, x) x-p F(z, x)}{x^{\mu}}$ uniformly for a.a. $z \in \Omega$;

(iv) there exist $\widehat{\delta}_{0}$ and $\widehat{C}_{0}>0$ such that $f(z, x) \geq \widehat{C}_{0} x^{q-1}$ for almost all $z \in \Omega$, all $x \in\left[0, \widehat{\delta}_{0}\right]$, with $q \in(1, p)$ as in hypothesis $\mathrm{H}(\mathrm{a})(\mathrm{v})$.

REMARKs 3.1. Since in this section we are looking for positive solutions and all the above hypotheses concern the positive half-axis $\mathbb{R}_{+}=[0, \infty)$, we may assume, without any loss of generality, that $f(z, x)=0$ for a.a. $z \in \Omega$, all $x \leq 0$.

Hypotheses $\left(\mathrm{H}_{1}\right)$ (ii)-(iii) imply that

$$
\lim _{x \rightarrow \infty} \frac{f(z, x)}{x^{p-1}}=\infty \quad \text { uniformly for a.a. } z \in \Omega,
$$

that is, for almost all $z \in \Omega, f(z, \cdot)$ is $(p-1)$-superlinear. Usually superlinear problems are treated using the so-called Ambrosetti-Rabinowitz condition (ARcondition, for short). We recall that the AR-condition (the unilateral version) says that there exist $\eta>p$ and $M>0$ such that

$$
0<\eta F(z, x) \leq f(z, x) x \quad \text { for a.a. } z \in \Omega,
$$

$$
\text { all } x \geq M \text { and } \operatorname{essinf} F(\cdot, M)>0 .
$$

From (3.1) through integration, we obtain the weaker condition

$$
C_{6} x^{\eta} \leq F(z, x) \text { for a.a. } z \in \Omega \text {, all } x \geq M \text { with } C_{6}>0 .
$$

By (3.2) and since $\eta>p$, we infer that the much weaker condition $\left(\mathrm{H}_{1}\right)$ (ii) holds.

Hypotheses $\left(\mathrm{H}_{1}\right)$ (ii)-(iii) together are weaker than the AR-condition and allow us to include in our framework superlinear functions with "slower" growth near $+\infty$ (see the examples below). 
Suppose that the AR-condition holds. We may assume that $\eta>\max \{(r-p)$ $N / p, 1\}$. We have

$$
\frac{f(z, x) x-p F(z, x)}{x^{\eta}}=\frac{f(z, x) x-\eta F(z, x)}{x^{\eta}}+(\eta-p) \frac{F(z, x)}{x^{\eta}} \geq(\eta-p) C_{6}
$$

for almost all $z \in \Omega$, all $x \geq M$. So, hypothesis $\left(\mathrm{H}_{1}\right)$ (iii) holds.

Hypothesis $\left(\mathrm{H}_{1}\right)$ (iv) implies that the reaction $f(z, \cdot)$ exhibits a concave term near zero. Therefore our hypotheses $\left(\mathrm{H}_{1}\right)$ incorporate the case of equations with competing nonlinearities ("concave-convex problems").

We mention that similar or different extensions of the AR-superlinearity condition can be found in Aizicovici, Papageorgiou and Staicu [3], Costa and Magalhães [8], Li and Yang [16], and Mugnai and Papageorgiou [22].

Example 3.2. The following functions satisfy hypotheses $\left(\mathrm{H}_{1}\right)$. For the sake of simplicity we drop the $z$-dependence:

$$
\begin{aligned}
& f_{1}(x)=x^{q-1}+x^{r-1} \quad \text { for all } x \geq 0 \text { with } 1<q<p<r<p^{*}, \\
& f_{2}(x)=\left\{\begin{array}{ll}
x^{q-1} & \text { if } x \in[0,1], \\
x^{p-1}(\ln x+1) & \text { if } 1<x,
\end{array} \text { with } 1<q<p .\right.
\end{aligned}
$$

Note that $f_{2}$ does not satisfy the AR-condition.

We introduce the following two sets: $\mathcal{L}=\left\{\lambda>0:\left(\mathrm{P}_{\lambda}\right)\right.$ admits a positive solution $\}$ and, for $\lambda \in \mathcal{L}, \mathcal{S}(\lambda)=\left\{\right.$ positive solutions of $\left.\left(\mathrm{P}_{\lambda}\right)\right\}$. We start with a useful observation concerning the solution set $\mathcal{S}(\lambda)$.

Proposition 3.3. If hypotheses $\mathrm{H}(\mathrm{a})(\mathrm{i})-$ (iii) and $\left(\mathrm{H}_{1}\right)$ hold, then

$$
\mathcal{S}(\lambda) \subseteq \operatorname{int} C_{+} .
$$

Proof. Let $\lambda \in \mathcal{L}$ and $u \in \mathcal{S}(\lambda)$. We have

$$
-\operatorname{div} a(D u(z))+\lambda u(z)^{p-1}=f(z, u(z)) \quad \text { a.e. in } \Omega, \frac{\partial u}{\partial n}=0 \text { on } \partial \Omega
$$

(see Motreanu and Papageorgiou [20]). From Hu and Papageorgiou [14] and Winkert [27], we have that $u \in L^{\infty}(\Omega)$. So, we can apply the regularity result of Lieberman [15] (p. 320) and infer that $u \in C_{+} \backslash\{0\}$.

Since $f \geq 0$ (see hypotheses $\left(\mathrm{H}_{1}\right)$ ), from (3.3) we have

$$
\operatorname{div} a(D u(z)) \leq \lambda u(z)^{p-1} \quad \text { for a.a. } z \in \Omega .
$$

Let $\chi(t)=t a_{0}(t)$ for all $t>0$. Then from the one-dimensional version of hypothesis $\mathrm{H}(\mathrm{a})$ (iii) we have

$$
t \chi^{\prime}(t)=t^{2} a_{0}^{\prime}(t)+t a_{0}(t) \geq C_{1} t^{p-1}
$$


hence

$$
\int_{0}^{t} s \chi^{\prime}(s) d s=t \chi(t)-\int_{0}^{t} \chi(s) d s=t^{2} a_{0}(t)-G_{0}(t) \geq \frac{C_{1}}{p} t^{p}
$$

for all $t \geq 0$. Let

$$
H(t)=t^{2} a_{0}(t)-G_{0}(t) \quad \text { and } \quad H_{0}(t)=\frac{C_{1}}{p} t^{p} \quad \text { for all } t \geq 0 .
$$

Let $s \in(0,1)$ and consider the sets

$$
D_{s}=\{t \in(0,1): H(t) \geq s\} \quad \text { and } \quad D_{s}^{0}=\left\{t \in(0,1): H_{0}(t) \geq s\right\} .
$$

From (3.5) we see that $D_{s}^{0} \subseteq D_{s}$, hence we have successively: $\inf D_{s}^{0} \leq \inf D_{s}$, $H^{-1}(s) \leq H_{0}^{-1}(s)$, and

$$
\int_{0}^{\delta} \frac{1}{H^{-1}\left(\lambda s^{p} / p\right)} d s \geq \int_{0}^{\delta} \frac{1}{H_{0}^{-1}\left(\lambda s^{p} / p\right)} d s=C_{7} \int_{0}^{\delta} \frac{d s}{s}=+\infty
$$

for some $C_{7}>0$. Because of (3.4) we can apply the strong maximum principle of Pucci and Serrin [25, p. 111] and deduce that $u(z)>0$ for all $z \in \Omega$. Then invoking the boundary point theorem of Pucci and Serrin [25, p. 120], we conclude that $u \in \operatorname{int} q C_{+}$. Therefore $\mathcal{S}(\lambda) \subseteq \operatorname{int} C_{+}$.

Next we show that $\mathcal{L}$ is nonempty and prove a structural property of $\mathcal{L}$, namely that $\mathcal{L}$ is a half-line.

Proposition 3.4. If hypotheses $\mathrm{H}(\mathrm{a})$ and $\left(\mathrm{H}_{1}\right)$ hold, then

$$
\mathcal{L} \neq \emptyset \text {, and } \lambda \in \mathcal{L} \text { implies that }[\lambda,+\infty) \subseteq \mathcal{L} .
$$

Proof. We consider the following auxiliary Neumann problem:

$$
-\operatorname{div} a(D u(z))+u(z)^{p-1}=1 \quad \text { in } \Omega, \quad \frac{\partial u}{\partial n}=0 \quad \text { on } \partial \Omega, \quad u>0 .
$$

Let $K_{p}: L^{p}(\Omega) \rightarrow L^{p^{\prime}}(\Omega)\left(1 / p+1 / p^{\prime}=1\right)$ be the nonlinear map defined by

$$
K_{p}(u)(\cdot)=|u(\cdot)|^{p-2} u(\cdot) \quad \text { for all } u \in L^{p}(\Omega) .
$$

Clearly $K_{p}$ is continuous and strictly monotone and so is $\left.K_{p}\right|_{W^{1, p}(\Omega)}$ which implies that $\left.K_{p}\right|_{W^{1, p}(\Omega)}$ is maximal monotone. Let $V: W^{1, p}(\Omega) \rightarrow W^{1, p}(\Omega)^{*}$ be defined by

$$
V(u)=A(u)+K_{p}(u) \text { for all } u \in W^{1, p}(\Omega) .
$$

Using Proposition 2.6, from Gasinski and Papageorgiou [11, p. 320], we conclude that $V$ is maximal monotone. Also, we have

$$
\begin{aligned}
\langle V(u), u\rangle & =\langle A(u), u\rangle+\|u\|_{p}^{p} \geq \frac{C_{1}}{p-1}\|D u\|_{p}^{p}+\|u\|_{p}^{p} & & \text { (see Lemma 2.3) } \\
& \geq C_{8}\|u\|^{p} & & \text { for some } C_{8}>0
\end{aligned}
$$


hence $V$ is coercive. Then from in $[11$, p. 320], we have that $V$ is surjective. So, we can find $\bar{u} \in W^{1, p}(\Omega), \bar{u} \neq 0$ such that $V(\bar{u})=0$, hence

$$
A(\bar{u})+|\bar{u}|^{p-2} \bar{u}=1 .
$$

On (3.7) we act with $-\bar{u}^{-}$and obtain

$$
\frac{C_{1}}{p-1}\left\|D \bar{u}^{-}\right\|_{p}^{p}+\left\|\bar{u}^{-}\right\|_{p}^{p} \leq 0
$$

(see Lemma 2.3), hence $\bar{u} \geq 0, \bar{u} \neq 0$. Then (3.7) becomes

$$
A(\bar{u})+\bar{u}^{p-1}=1,
$$

hence $\bar{u}$ is a positive solution of the auxiliary problem (3.6).

As in the proof of Proposition 3.3, using the nonlinear regularity theory (see [14], [27] and [15]) and the nonlinear maximum principle (see [25]), we have $\bar{u} \in \operatorname{int} C_{+}$. So, we can find $C_{9}>0$ such that $\bar{u}(z) \geq C_{9}$ for all $z \in \bar{\Omega}$. Let

$$
\lambda_{0}=\frac{1+\left\|N_{f}(\bar{u})\right\|_{\infty}}{C_{9}^{p-1}}
$$

(see hypothesis $\left.\left(\mathrm{H}_{1}\right)(\mathrm{i})\right)$. Then

$$
A(\bar{u})+\lambda_{0} \bar{u}^{p-1} \geq N_{f}(\bar{u}) \quad \text { in } W^{1, p}(\Omega)^{*} .
$$

Using $\bar{u} \in \operatorname{int} C_{+}$, we introduce the following truncation of the reaction $f(z, \cdot)$ :

$$
f_{0}(z, x)= \begin{cases}0 & \text { if } x<0 \\ f(z, x) & \text { if } 0 \leq x \leq \bar{u}(z) \\ f(z, \bar{u}(z)) & \text { if } \bar{u}(z)<x\end{cases}
$$

This is a Carathéodory function. Let

$$
F_{0}(z, x)=\int_{0}^{x} f_{0}(z, s) d s
$$

and consider the $C^{1}$-functional $\varphi_{0}: W^{1, p}(\Omega) \rightarrow \mathbb{R}$ defined by

$$
\varphi_{0}(u)=\int_{\Omega} G(D u(z)) d z+\frac{\lambda_{0}}{p}\|u\|_{p}^{p}-\int_{\Omega} F_{0}(z, u(z)) d z \text { for all } u \in W^{1, p}(\Omega) .
$$

From (3.9) it is clear that $\varphi_{0}$ is coercive. Also, using the Sobolev embedding theorem, we see that $\varphi_{0}$ is sequentially weakly lower semicontinuous. So, by the Weierstrass theorem, we can find $u_{0} \in W^{1, p}(\Omega)$ such that

$$
\varphi_{0}\left(u_{0}\right)=\inf \left\{\varphi_{0}(u): u \in W^{1, p}(\Omega)\right\} .
$$

By virtue of $\mathrm{H}(\mathrm{a})(\mathrm{v})$ and $\left(\mathrm{H}_{1}\right)(\mathrm{iv})$, given $\varepsilon>0$, we can find $\delta=\delta(\varepsilon) \in\left(0, \widehat{\delta}_{0}\right]$ such that

$$
G_{0}(t) \leq \frac{\varepsilon}{q} t^{q} \quad \text { for all } t \in[0, \delta],
$$


hence

$$
G(y) \leq \frac{\varepsilon}{q}\|y\|^{q} \quad \text { for all } y \in \mathbb{R}^{N} \text { with }\|y\| \leq \delta .
$$

Given $u \in \operatorname{int} C_{+}$, we can find $t \in(0,1]$ small enough such that

$$
t u \leq \bar{u}, \quad t u(z) \in(0, \delta] \quad \text { and } \quad t\|D u(z)\| \in[0, \delta] \quad \text { for all } z \in \bar{\Omega}
$$

(recall $u, \bar{u} \in \operatorname{int} C_{+}$and use Lemma 3.3 of Filippakis, Kristaly and Papageorgiou [10]). Then we have

$$
\begin{aligned}
\varphi_{0}(t u) & =\int_{\Omega} G(t D u(z)) d z+\frac{\lambda_{0} t^{p}}{p}\|u\|_{p}^{p}-\int_{\Omega} F_{0}(z, t u(z)) d z \\
& \leq \frac{\lambda_{0} t^{p}}{p}\|u\|_{p}^{p}-\frac{t^{q}}{q}\left[\widehat{C}_{0}\|u\|_{q}^{q}-\varepsilon\|D u\|_{q}^{q}\right]
\end{aligned}
$$

(see (3.11), (3.12) and hypothesis $\mathrm{H}(\mathrm{a})(\mathrm{iv}))$. We choose $\varepsilon \in\left(0, \widehat{C}_{0}\|u\|_{q}^{q} /\|D u\|_{q}^{q}\right)$. Then from (3.13) it follows

$$
\varphi_{0}(t u) \leq \frac{\lambda_{0} t^{p}}{p}\|u\|_{p}^{p}-C_{10} t^{q} \quad \text { for some } C_{10}=C_{10}(u)>0 .
$$

Since $q<p$ (see hypothesis $\mathrm{H}(\mathrm{a})$ (iv) ), choosing $t \in(0,1)$ even smaller if necessary, from $(3.14)$ we see that $\varphi_{0}(t u)<0$ which implies $\varphi_{0}\left(u_{0}\right)<0=\varphi_{0}(0)$ (see (3.10)), hence $u_{0} \neq 0$.

From (3.10) we have $\varphi_{0}^{\prime}\left(u_{0}\right)=0$, hence

$$
A\left(u_{0}\right)+\lambda_{0}\left|u_{0}\right|^{p-2} u_{0}=N_{f_{0}}\left(u_{0}\right) .
$$

On (3.15) we act with $-u_{0}^{-} \in W^{1, p}(\Omega)$ and obtain

$$
\frac{C_{1}}{p-1}\left\|D u_{0}^{-}\right\|_{p}^{p}+\lambda_{0}\left\|u_{0}^{-}\right\|_{p}^{p} \leq 0 \quad \text { (see Lemma } 2.3 \text { and (3.9)), }
$$

hence $u_{0} \geq 0, u_{0} \neq 0$. Also, on $(3.15)$ we act with $\left(u_{0}-\bar{u}\right)^{+} \in W^{1, p}(\Omega)$. We obtain

$$
\begin{aligned}
\left\langle A\left(u_{0}\right),\right. & \left.\left(u_{0}-\bar{u}\right)^{+}\right\rangle+\lambda_{0} \int_{\Omega} u_{0}^{p-1}\left(u_{0}-\bar{u}\right)^{+} d z \\
& =\int_{\Omega} f_{0}\left(z, u_{0}\right)\left(u_{0}-\bar{u}\right)^{+} d z \\
& =\int_{\Omega} f(z, \bar{u})\left(u_{0}-\bar{u}\right)^{+} d z \\
& \leq\left\langle A(\bar{u}),\left(u_{0}-\bar{u}\right)^{+}\right\rangle+\lambda_{0} \int_{\Omega} \bar{u}^{p-1}\left(u_{0}-\bar{u}\right)^{+} d z \quad(\text { see }(3.9))
\end{aligned}
$$

hence

$$
\begin{aligned}
\int_{\left\{u_{0}>\bar{u}\right\}}\left(a\left(D u_{0}\right)-a(D \bar{u}), D u_{0}\right. & -D \bar{u})_{\mathbb{R}^{N}} \\
& +\lambda_{0} \int_{\left\{u_{0}>\bar{u}\right\}}\left(u_{0}^{p-1}-\bar{u}^{p-1}\right)\left(u_{0}-\bar{u}\right) d z \leq 0,
\end{aligned}
$$


therefore $\left|\left\{u_{0}>\bar{u}\right\}\right|_{N}=0$, and we conclude that $u_{0} \leq \bar{u}$. So, we have proved that

$$
u_{0} \in[0, \bar{u}]:=\left\{u \in W^{1, p}(\Omega): 0 \leq u(z) \leq \bar{u}(z) \text { for a.a. } z \in \Omega\right\}, \quad u_{0} \neq 0 .
$$

Then equation (3.15) becomes

$$
A\left(u_{0}\right)+\lambda_{0} u_{0}^{p-1}=N_{f}\left(u_{0}\right)
$$

(see (3.9)) therefore $u_{0} \in \mathcal{S}\left(\lambda_{0}\right) \subseteq \operatorname{int} C_{+}$(see Proposition 3.3) and so $\lambda_{0} \in \mathcal{L}$.

Now, let $\lambda \in \mathcal{L}$ and $\eta>\lambda$. Then there exists $u_{\lambda} \in \mathcal{S}(\lambda) \subseteq \operatorname{int} C_{+}$(see Proposition 3.3). We have

$$
A\left(u_{\lambda}\right)+\eta u_{\lambda}^{p-1} \geq A\left(u_{\lambda}\right)+\lambda u_{\lambda}^{p-1}=N_{f}\left(u_{\lambda}\right) \text { in } W^{1, p}(\Omega)^{*} .
$$

We truncate $f(z, \cdot)$ at $u_{\lambda}(z)$ and, reasoning as above with $\bar{u}$ replaced by $u_{\lambda}$ and using (3.16) instead of (3.8), via the direct method, we produce

$$
u_{\eta} \in\left[0, u_{\lambda}\right] \cap \mathcal{S}(\eta) \subseteq\left[0, u_{\lambda}\right] \cap \operatorname{int} C_{+} .
$$

Therefore $\eta \in \mathcal{L}$ and so we conclude that $[\lambda,+\infty) \subseteq \mathcal{L}$.

A useful by-product of the above proof is the following corollary:

Corollary 3.5. If hypotheses $\mathrm{H}(\mathrm{a})$ and $\left(\mathrm{H}_{1}\right)$ hold, $\eta>\lambda \in \mathcal{L}$ and $u_{\lambda} \in$ $\mathcal{S}(\lambda) \subseteq \operatorname{int} C_{+}$, then we can find $u_{\eta} \in \mathcal{S}(\eta) \subseteq \operatorname{int} C_{+}$such that $u_{\eta} \leq u_{\lambda}$.

In fact, we can improve the conclusion of this corollary provided we strengthen a little the hypotheses on the reaction $f$. The new hypotheses on the reaction $f$ are the following:

$\left(\mathrm{H}_{2}\right) f: \Omega \times \mathbb{R} \rightarrow \mathbb{R}$ is a Carathéodory function such that for almost all $z \in \Omega$ $f(z, 0)=0, f(z, x)>0$ for all $x>0$, hypotheses $\left(\mathrm{H}_{2}\right)$ (i)-(iv) are the same as $\left(\mathrm{H}_{1}\right)(\mathrm{i})-(\mathrm{iv})$ and

(v) for every $\rho>0$, there exists $\xi_{\rho}>0$ such that for almost all $z \in \Omega$ the function $x \mapsto f(z, x)+\xi_{\rho} x^{p-1}$ is nondecreasing on $[0, \rho]$.

Remark 3.6. Note that if for almost all $z \in \Omega, f(z, \cdot) \in C^{1}(0, \infty)$ and $f_{x}(z, \cdot)$ is $L^{\infty}(\Omega)$-bounded on compact subsets of $(0, \infty)$, hypothesis $\left(\mathrm{H}_{2}\right)(\mathrm{v})$ is automatically satisfied. So, the two examples given after hypotheses $\left(\mathrm{H}_{1}\right)$ satisfy $\left(\mathrm{H}_{2}\right)(\mathrm{v})$.

Proposition 3.7. If hypotheses $\mathrm{H}(\mathrm{a})$ and $\left(\mathrm{H}_{2}\right)$ hold, $\eta>\lambda \in \mathcal{L}$ and $u_{\lambda} \in$ $\mathcal{S}(\lambda) \subseteq \operatorname{int} C_{+}$, then we can find $u_{\eta} \in \mathcal{S}(\eta) \subseteq \operatorname{int} C_{+}$such that $u_{\lambda}-u_{\eta} \in \operatorname{int} C_{+}$.

Proof. From Corollary 3.5, we know that there exists $u_{\eta} \in \mathcal{S}(\eta) \subseteq \operatorname{int} C_{+}$ such that

$$
u_{\eta} \leq u_{\lambda}
$$


Let $\delta>0$ and set $u_{\eta}^{\delta}=u_{\eta}+\delta \in \operatorname{int} C_{+}$. Let $\rho=\left\|u_{\lambda}\right\|_{\infty}$ and let $\xi_{\rho}>0$ be as postulated by hypothesis $\left(\mathrm{H}_{2}\right)(\mathrm{v})$. We have

$$
\begin{array}{rlr}
-\operatorname{div} a\left(D u_{\eta}^{\delta}\right)+\left(\lambda+\xi_{\rho}\right)\left(u_{\eta}^{\delta}\right)^{p-1} & \\
\leq-\operatorname{div} a\left(D u_{\eta}\right)+\eta u_{\eta}^{p-1}-(\eta-\lambda) u_{\eta}^{p-1}+\xi_{\rho} u_{\eta}^{p-1}+\sigma(\delta) \\
& & \left(\operatorname{with} \sigma(\delta) \rightarrow 0^{+} \text {as } \delta \rightarrow 0^{+}\right) \\
\leq-\operatorname{div} a\left(D u_{\eta}\right)+\left(\eta+\xi_{\rho}\right) u_{\eta}^{p-1}- & (\eta-\lambda) m_{\eta}^{p-1}+\sigma(\delta) \\
& & \left(\text { with } m_{\eta}=\min _{\bar{\Omega}} u_{\eta}>0\right) \\
\leq-\operatorname{div} a\left(D u_{\eta}\right)+\left(\eta+\xi_{\rho}\right) u_{\eta}^{p-1} & & (\text { for } \delta>0 \text { small }) \\
=f\left(z, u_{\eta}\right)+\xi_{\rho} u_{\eta}^{p-1} & & \left(\text { since } u_{\eta} \in \mathcal{S}(\eta)\right) \\
\leq f\left(z, u_{\lambda}\right)+\xi_{\rho} u_{\lambda}^{p-1} & & \left(\text { see }(3.17) \text { and hypothesis }\left(\mathrm{H}_{2}\right)(\mathrm{v})\right) \\
=-\operatorname{div} a\left(D u_{\lambda}\right)+\xi_{\rho} u_{\lambda}^{p-1} & & \left(\text { since } u_{\lambda} \in \mathcal{S}(\lambda)\right),
\end{array}
$$

hence $u_{\eta}^{\delta} \leq u_{\lambda}$ for all $\delta>0$ small (see Damascelli [9, p. 495]) therefore $u_{\lambda}-u_{\eta}$ lies in int $C_{+}$.

Let $\lambda_{*}=\inf \mathcal{L}$. In what follows, for every $\lambda>0, \varphi_{\lambda}: W^{1, p}(\Omega) \rightarrow \mathbb{R}$ is the energy functional defined by

$$
\varphi_{\lambda}(u)=\int_{\Omega} G(D u(z)) d z+\frac{\lambda}{p}\|u\|_{p}^{p}-\int_{\Omega} F(z, u(z)) d z \quad \text { for all } u \in W^{1, p}(\Omega) .
$$

Evidently $\varphi_{\lambda} \in C^{1}\left(W^{1, p}(\Omega)\right)$.

Proposition 3.8. If hypotheses $\mathrm{H}(\mathrm{a})$ and $\left(\mathrm{H}_{1}\right)$ hold, then $\lambda_{*}>0$.

Proof. We argue by contradiction. So, suppose that $\lambda_{*}=0$ and let $\left\{\lambda_{n}\right\}_{n \in \mathbb{N}} \subseteq(0, \infty) \subseteq \mathcal{L}$ be such that $\lambda_{n} \downarrow 0$ as $n \rightarrow \infty$. We can find $u_{n} \in \mathcal{S}\left(\lambda_{n}\right)$ for $n \geq 1$, such that $\left\{u_{n}\right\}_{n \in \mathbb{N}}$ is nondecreasing and

$$
\varphi_{\lambda_{n}}\left(u_{n}\right)<0 \text { for all } n \geq 1
$$

(see the last part of the proof of Proposition 3.4). From (3.18) we have

$$
-\int_{\Omega} p F\left(z, u_{n}\right) d z \leq-\int_{\Omega} p G\left(D u_{n}\right) d z-\lambda_{n}\left\|u_{n}\right\|_{p}^{p} \quad \text { for all } n \geq 1 .
$$

Since $u_{n} \in \mathcal{S}\left(\lambda_{n}\right)$ for $n \geq 1$, we have $A\left(u_{n}\right)+\lambda_{n} u_{n}^{p-1}=N_{f}\left(u_{n}\right)$ hence

$$
\int_{\Omega} f\left(z, u_{n}\right) u_{n} d z=\int_{\Omega}\left(a\left(D u_{n}\right), D u_{n}\right)_{\mathbb{R}^{N}}+\lambda_{n}\left\|u_{n}\right\|_{p}^{p} \quad \text { for all } n \geq 1 .
$$

Adding (3.19) and (3.20), we obtain

$$
\int_{\Omega}\left[f\left(z, u_{n}\right) u_{n}-p F\left(z, u_{n}\right)\right] d z \leq \int_{\Omega}\left[\left(a\left(D u_{n}\right), D u_{n}\right)_{\mathbb{R}^{N}}-p G\left(D u_{n}\right)\right] d z,
$$

hence

$$
\int_{\Omega}\left[f\left(z, u_{n}\right) u_{n}-p F\left(z, u_{n}\right)\right] d z \leq \xi_{0} \quad \text { for all } n \geq 1(\text { see H(a) (iv)). }
$$


From hypotheses $\left(\mathrm{H}_{1}\right)(\mathrm{i})$, (iii), we see that we can find $\beta_{1} \in\left(0, \beta_{0}\right)$ and $C_{11}>0$ such that

$$
\beta_{1} x^{\mu}-C_{11} \leq f(z, x) x-p F(z, x) \text { for a.a. } z \in \Omega \text {, all } x \geq 0 .
$$

Using (3.21) and (3.20), we have $\left\|u_{n}\right\|_{\mu}^{\mu} \leq C_{12}$ for some $C_{12}>0$ and all $n \geq 1$, hence

$$
\left\{u_{n}\right\}_{n \in \mathbb{N}} \subseteq L^{\mu}(\Omega) \text { is bounded. }
$$

First suppose that $p<N$. It is clear from hypothesis $\left(\mathrm{H}_{1}\right)$ (iii) that without any lost of generality we may assume that $\mu<r<p^{*}$. Let $t \in(0,1)$ such that

$$
\frac{1}{r}=\frac{1-t}{\mu}+\frac{t}{p^{*}} .
$$

Invoking the interpolation inequality (see, for example, Gasinski and Papageorgiou $[11$, p. 905]), we have

$$
\left\|u_{n}\right\|_{r} \leq\left\|u_{n}\right\|_{\mu}^{1-t}\left\|u_{n}\right\|_{p^{*}}^{t} \text { for all } n \geq 1 .
$$

Then using (3.22) and the Sobolev embedding theorem, we have

$$
\left\|u_{n}\right\|_{r}^{r} \leq C_{13}\left\|u_{n}\right\|^{t r} \quad \text { for some } C_{13}>0 \text {, all } n \geq 1 .
$$

Hypothesis $\left(\mathrm{H}_{1}\right)(\mathrm{i})$ implies that

$$
f(z, x) \leq C_{14}\left(1+x^{r}\right) \quad \text { for a.a. } z \in \Omega \text {, all } x \geq 0 \text {, some } C_{14}>0 .
$$

From (3.20) and (3.25), we have

$$
\int_{\Omega}\left(a\left(D u_{n}\right), D u_{n}\right)_{\mathbb{R}^{N}}+\lambda_{n}\left\|u_{n}\right\|_{p}^{p} \leq C_{15}\left(1+\left\|u_{n}\right\|_{r}^{r}\right)
$$

for some $C_{15}>0$, all $n \geq 1$, hence

$$
\frac{C_{1}}{p-1}\left\|D u_{n}\right\|_{p}^{p} \leq C_{16}\left(1+\left\|u_{n}\right\|^{t r}\right) \quad \text { for some } C_{16}>0 \text {, all } n \geq 1 \text {, }
$$

(see Lemma 2.3 and (3.24)). Recall that $u \rightarrow\|u\|_{\mu}+\|D u\|_{p}$ is an equivalent norm on the Sobolev space $W^{1, p}(\Omega)$ (see, for example, Gasinski and Papageorgiou [11, p. 227]). So, from (3.22) and (3.26) we have

$$
\left\|u_{n}\right\|^{p} \leq C_{17}\left(1+\left\|u_{n}\right\|^{t r}\right) \text { for some } C_{17}>0 \text {, all } n \geq 1 .
$$

From (3.23) and the hypothesis on $\mu$ (see $\left(\mathrm{H}_{1}\right)$ (iii)) it follows that $t r<p$. Therefore from (3.27), we infer that

$$
\left\{u_{n}\right\}_{n \in \mathbb{N}} \subseteq W^{1, p}(\Omega) \text { is bounded. }
$$

If $p \geq N$, then $p^{*}=+\infty$ and $W^{1, p}(\Omega) \hookrightarrow L^{\theta}(\Omega)$ for all $\theta \in[1,+\infty)$. Then the previous argument works if we replace $p^{*}$ by $\eta>r>\mu$ and we choose $t \in(0,1)$ such that

$$
\frac{1}{r}=\frac{1-t}{\mu}+\frac{t}{\eta},
$$


that is,

$$
t r=\frac{\eta(r-\mu)}{\eta-\mu} .
$$

Note that $\eta(r-\mu) /(\eta-\mu) \rightarrow r-\mu$ as $\eta \rightarrow+\infty=p^{*}$. But by hypothesis $\left(\mathrm{H}_{1}\right)$ (iii), $r-\mu<p$. Therefore for $\eta>r$ large, we have $t r<p$ and so again (3.28) holds.

By virtue of (3.28) and by passing to a subsequence if necessary, we may assume that

$$
u_{n} \stackrel{\mathrm{w}}{\longrightarrow} u \quad \text { in } W^{1, p}(\Omega) \quad \text { and } \quad u_{n} \rightarrow u \quad \text { in } L^{r}(\Omega) \quad \text { as } n \rightarrow \infty .
$$

Recall that

$$
A\left(u_{n}\right)+\lambda_{n} u_{n}^{p-1}=N_{f}\left(u_{n}\right) \text { for all } n \geq 1 .
$$

On (3.30) we act with $u_{n}-u \in W^{1, p}(\Omega)$, pass to the limit as $n \rightarrow \infty$ and use (3.29) to obtain $\lim _{n \rightarrow \infty}\left\langle A\left(u_{n}\right), u_{n}-u\right\rangle=0$, hence

$$
u_{n} \rightarrow u \quad \text { in } W^{1, p}(\Omega) \text { as } n \rightarrow \infty
$$

(see Proposition 2.6). So, if in (3.30) we pass to the limit as $n \rightarrow \infty$ and use (3.31) and the fact that $\lambda_{n} \downarrow 0$, we obtain

$$
A(u)=N_{f}(u) .
$$

By the nonlinear regularity theory (see Lieberman [15]) it follows that $u \in C_{+}$.

Claim. $u \neq 0$.

From hypotheses $\left(\mathrm{H}_{1}\right)(\mathrm{i})(\mathrm{iv})$, we see that we can find $C_{18}>0$ such that

$$
f(z, x) \geq \widehat{C}_{0} x^{q-1}-C_{18} x^{r-1} \quad \text { for a.a. } z \in \Omega, \text { all } x \geq 0 .
$$

Motivated by this unilateral growth estimate, we introduce the following auxiliary Neumann problem:

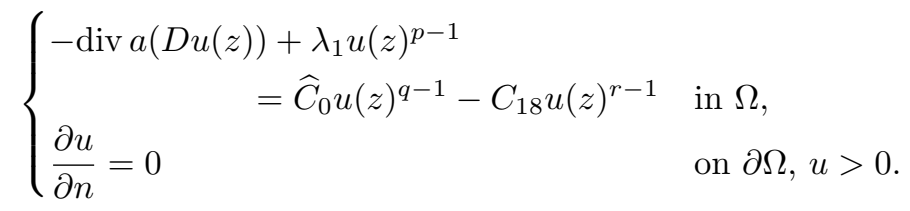

Let $\psi: W^{1, p}(\Omega) \rightarrow \mathbb{R}$ be the energy functional for problem (3.33) defined by

$$
\psi(u)=\int_{\Omega} G(D u(z)) s d z+\frac{\lambda_{1}}{p}\|u\|_{p}^{p}-\frac{\widehat{C}_{0}}{q}\left\|u^{+}\right\|_{q}^{q}+\frac{C_{18}}{r}\left\|u^{+}\right\|_{r}^{r}
$$

for all $u \in W^{1, p}(\Omega)$. Since $q<p<r$, it is clear that $\psi$ is coercive (see Corollary 2.4). Also, by the Sobolev embedding theorem, we see that $\psi$ is sequentially weakly lower semicontinuous. So, we can find $\widetilde{u} \in W^{1, p}(\Omega)$ such that

$$
\psi(\widetilde{u})=\inf \left\{\psi(u): u \in W^{1, p}(\Omega)\right\} .
$$


Because $q<p<r$, as in the proof of Proposition 3.4, we check that $\psi(\widetilde{u})<0=$ $\psi(u)$, hence $\widetilde{u} \neq 0$. From (3.34), we have $\psi^{\prime}(\widetilde{u})=0$ hence

$$
A(\widetilde{u})+\lambda_{1}|\widetilde{u}|^{p-2} \widetilde{u}=\widehat{C}_{0}\left(\widetilde{u}^{+}\right)^{q-1}-C_{18}\left(\widetilde{u}^{+}\right)^{r-1} .
$$

On (3.35) we act with $-\widetilde{u}^{-} \in W^{1, p}(\Omega)$, and using Lemma 2.3 , we obtain $\widetilde{u} \geq 0$, $\widetilde{u} \neq 0$. Then (3.35) becomes

$$
A(\widetilde{u})+\lambda_{1} \widetilde{u}^{p-1}=\widehat{C}_{0} \widetilde{u}^{q-1}-C_{18} \widetilde{u}^{r-1},
$$

hence $\widetilde{u}$ is a positive solution of (3.33) and $\widetilde{u} \in \operatorname{int} C_{+}$(by nonlinear regularity [15] and the nonlinear maximum principle [25]). Moreover, as in Aizicovici, Papageorgiou and Staicu [4], we conclude that $\widetilde{u} \in \operatorname{int} C_{+}$is the unique positive solution of (3.33).

Let $u_{1} \in \mathcal{S}\left(\lambda_{1}\right) \subseteq \operatorname{int} C_{+}$and consider the Carathéodory function

$$
k(z, x)= \begin{cases}0 & \text { if } x<0, \\ \widehat{C}_{0} x^{q-1}-C_{18} x^{r-1} & \text { if } 0 \leq x \leq u_{1}(z), \\ \widehat{C}_{0} u_{1}(z)^{q-1}-C_{18} u_{1}(z)^{r-1} & \text { if } u_{1}(z)<x .\end{cases}
$$

We set

$$
K(z, x)=\int_{0}^{x} k(z, s) d s
$$

and consider the $C^{1}$-functional $\widehat{\gamma}: W^{1, p}(\Omega) \rightarrow \mathbb{R}$ defined by

$$
\widehat{\gamma}(u)=\int_{\Omega} G(D u(z)) d z+\frac{\lambda_{1}}{p}\|u\|_{p}^{p}-\int_{\Omega} K(z, u(z)) d z \quad \text { for all } u \in W^{1, p}(\Omega) .
$$

From (3.36) it is clear that $\widehat{\gamma}$ is coercive. Also, it is sequentially weakly lower semicontinuous. So, we can find $\widetilde{u}_{0} \in W^{1, p}(\Omega)$ such that

$$
\widehat{\gamma}\left(\widetilde{u}_{0}\right)=\inf \left\{\widehat{\gamma}(u): u \in W^{1, p}(\Omega)\right\} .
$$

As before (see the proof of Proposition 3.4), since $1<q<p<r$, we have $\widehat{\gamma}\left(\widetilde{u}_{0}\right)<0=\widehat{\gamma}(0)$, hence $\widetilde{u}_{0} \neq 0$. From $(3.37)$, we have $\widehat{\gamma}^{\prime}\left(\widetilde{u}_{0}\right)=0$ hence

$$
A\left(\widetilde{u}_{0}\right)+\lambda_{1}\left|\widetilde{u}_{0}\right|^{p-2} \widetilde{u}_{0}=N_{k}\left(\widetilde{u}_{0}\right) .
$$

On (3.38) we first act with $-\widetilde{u}_{0}^{-} \in W^{1, p}(\Omega)$ and then with $\left(\widetilde{u}_{0}-u_{1}\right)^{+} \in W^{1, p}(\Omega)$ and obtain

$$
\widetilde{u}_{0} \in\left[0, u_{1}\right]:=\left\{u \in W^{1, p}(\Omega): 0 \leq u(z) \leq u_{1}(z) \text { for a.a. } z \in \Omega\right\}
$$

(see the proof of Proposition 3.4). Using (3.36) and (3.37) we obtain

$$
A\left(\widetilde{u}_{0}\right)+\lambda_{1} \widetilde{u}_{0}^{p-1}=\widehat{C}_{0} \widetilde{u}_{0}^{q-1}-C_{18} \widetilde{u}_{0}^{r-1},
$$

hence $\widetilde{u}_{0}$ is a positive solution of (3.33), and by the uniqueness of the positive solution of (3.33), it follows that $\widetilde{u}_{0}=\widetilde{u} \in \operatorname{int} C_{+}$. So, we can say that $\widetilde{u} \leq u_{1} \leq$ $u_{n}$ for all $n \geq 1$ (recall that $\left\{u_{n}\right\}_{n \in \mathbb{N}}$ is nondecreasing), hence $\widetilde{u} \leq u$ (see (3.31)), therefore $u \neq 0$. This proves Claim. 
On (3.32) we act with $1 \in \operatorname{int} C_{+}$. We obtain

$$
0=\int_{\Omega} f(z, u) d z
$$

But our hypotheses on $f$ and the Claim, imply $\int_{\Omega} f(z, u) d z>0$, a contradiction. This means that $\lambda_{*}>0$.

If we use the stronger hypotheses $\left(\mathrm{H}_{2}\right)$, we can show that for $\lambda \in\left(\lambda_{*}, \infty\right)$, problem $\left(\mathrm{P}_{\lambda}\right)$ admits at least two positive solutions.

Proposition 3.9. If hypotheses $\mathrm{H}(\mathrm{a})$ and $\left(\mathrm{H}_{2}\right)$ hold and $\lambda \in\left(\lambda_{*}, \infty\right)$, then $\left(\mathrm{P}_{\lambda}\right)$ admits at least two positive solutionsu $u_{\lambda}, \widehat{u}_{\lambda} \in \operatorname{int} C_{+}, u_{\lambda} \neq \widehat{u}_{\lambda}$.

Proof. Let $\eta_{1}, \eta_{2} \in \mathcal{L}$ and assume that $\lambda_{*}<\eta_{1}<\lambda<\eta_{2}$. From Proposition 3.7, we know that we can find $u_{\eta_{1}} \in \mathcal{S}\left(\eta_{1}\right) \subseteq \operatorname{int} C_{+}$and $u_{\eta_{2}} \in \mathcal{S}\left(\eta_{2}\right) \subseteq$ $\operatorname{int} C_{+}$such that $u_{\eta_{1}}-u_{\eta_{2}} \in \operatorname{int} C_{+}$.

We introduce the following Carathéodory function:

$$
w(z, x)= \begin{cases}f\left(z, u_{\eta_{2}}(z)\right) & \text { if } x<u_{\eta_{2}}(z), \\ f(z, x) & \text { if } u_{\eta_{2}}(z) \leq x \leq u_{\eta_{1}}(z), \\ f\left(z, u_{\eta_{1}}(z)\right) & \text { if } u_{\eta_{1}}(z)<x .\end{cases}
$$

We set

$$
W(z, x)=\int_{0}^{x} w(z, s) d s
$$

and consider the $C^{1}$-functional $\xi_{\lambda}: W^{1, p}(\Omega) \rightarrow \mathbb{R}$ defined by

$$
\xi_{\lambda}(u)=\int_{\Omega} G(D u(z)) d z+\frac{\lambda}{p}\|u\|_{p}^{p}-\int_{\Omega} W(z, u(z)) d z \quad \text { for all } u \in W^{1, p}(\Omega) .
$$

From (3.39) we see that $\xi_{\lambda}$ is coercive. Also, it is sequentially weakly lower semicontinuous. So, we can find $u_{\lambda} \in W^{1, p}(\Omega)$ such that

$$
\xi_{\lambda}\left(u_{\lambda}\right)=\inf \left\{\xi_{\lambda}(u): u \in W^{1, p}(\Omega)\right\}
$$

hence $\xi_{\lambda}^{\prime}\left(u_{\lambda}\right)=0$ therefore

$$
A\left(u_{\lambda}\right)+\lambda\left|u_{\lambda}\right|^{p-2} u_{\lambda}=N_{w}\left(u_{\lambda}\right) .
$$

On (3.40) we act with $\left(u_{\lambda}-u_{\eta_{1}}\right)^{+} \in W^{1, p}(\Omega)$ and with $\left(u_{\eta_{2}}-u_{\lambda}\right)^{+} \in W^{1, p}(\Omega)$, and obtain

$$
u_{\lambda} \in\left[u_{\eta_{2}}, u_{\eta_{1}}\right]:=\left\{u \in W^{1, p}(\Omega): u_{\eta_{2}}(z) \leq u(z) \leq u_{\eta_{1}}(z) \text { for a.a. } z \in \Omega\right\} .
$$

In fact, reasoning as in the proof of Proposition 3.7, we show that

$$
u_{\lambda}-u_{\eta_{2}} \in \operatorname{int} C_{+} \text {and } u_{\eta_{1}}-u_{\lambda} \in \operatorname{int} C_{+},
$$

hence

$$
u_{\lambda}(z) \in \operatorname{int}_{C^{1}(\bar{\Omega})}\left[u_{\eta_{2}}, u_{\eta_{1}}\right]
$$


Then from (3.39) we see that $u_{\lambda} \in \mathcal{S}(\lambda) \subseteq \operatorname{int} C_{+}$. So, we have produced one positive solution for $\left(\mathrm{P}_{\lambda}\right)$. To produce a second positive solution, we introduce the Carathéodory function $\widehat{k}$ defined by

$$
\widehat{k}(z, x)= \begin{cases}f\left(z, u_{\eta_{2}}(z)\right) & \text { if } x<u_{\eta_{2}}(z), \\ f(z, x) & \text { if } u_{\eta_{2}}(z) \leq x .\end{cases}
$$

Let

$$
\widehat{K}(z, x)=\int_{0}^{x} k(z, s) d s
$$

and consider the $C^{1}$-functional $\sigma_{\lambda}: W^{1, p}(\Omega) \rightarrow \mathbb{R}$ defined by

$$
\widehat{\sigma}_{\lambda}(u)=\int_{\Omega} G(D u(z)) d z+\frac{\lambda}{p}\|u\|_{p}^{p}-\int_{\Omega} \widehat{K}(z, u(z)) d z \quad \text { for all } u \in W^{1, p}(\Omega) .
$$

As before (see the proof of Proposition 3.4) we can check that

$$
K_{\widehat{\sigma}_{\lambda}} \subseteq\left[u_{\eta_{2}}\right):=\left\{u \in W^{1, p}(\Omega): u_{\eta_{2}}(z) \leq u(z) \text { for a.a. } z \in \Omega\right\} .
$$

From (3.39) and (3.42) we see

$$
\left.\xi_{\lambda}\right|_{\left[u_{\eta_{2}}, u_{\eta_{1}}\right]}=\left.\widehat{\sigma}_{\lambda}\right|_{\left[u_{\eta_{2}}, u_{\eta_{1}}\right]} \cdot
$$

By (3.41) and (3.44) and since $u_{\lambda}$ is a minimizer of $\xi_{\lambda}$, it follows that $u_{\lambda}$ is a $C^{1}(\bar{\Omega})$-minimizer of $\widehat{\sigma}_{\lambda}$. Invoking Proposition 2.7, we infer that $u_{\lambda}$ is a $W^{1, p}(\Omega)$ minimizer of $\widehat{\sigma}_{\lambda}$.

We may assume that $K_{\widehat{\sigma}_{\lambda}}$ is finite or otherwise we have an infinity of positive solutions for problem $\left(\mathrm{P}_{\lambda}\right)$ (see (3.43) and (3.42)). Then, from Aizicovici, Papageorgiou and Staicu [1] (see the proof of Proposition 29), we can find $\rho \in(0,1)$ small such that

$$
\widehat{\sigma}_{\lambda}\left(u_{\lambda}\right)<\inf \left\{\widehat{\sigma}_{\lambda}(u):\left\|u-u_{\lambda}\right\|=\rho\right\}=: \widehat{m}_{\lambda} .
$$

Hypothesis $\left(\mathrm{H}_{2}\right)$ (ii) implies

$$
\widehat{\sigma}_{\lambda}(\xi) \rightarrow-\infty \quad \text { as } \xi \rightarrow+\infty, \xi \in \mathbb{R} .
$$

In addition, minor changes in the first part of the proof of Proposition 3.8, reveal that

$$
\widehat{\sigma}_{\lambda} \text { satisfies the C-condition }
$$

(see also Aizicovici, Papageorgiou and Staicu [3]). Then (3.45)-(3.47) permit the use of Theorem 2.1 (the mountain pass theorem). So, we can find $\widehat{u}_{\lambda} \in W^{1, p}(\Omega)$ such that

$$
\widehat{u}_{\lambda} \in K_{\widehat{\sigma}_{\lambda}} \subseteq\left[u_{\eta_{2}}\right) \quad\left(\text { see (3.43)) } \quad \text { and } \quad \widehat{\sigma}_{\lambda}\left(u_{\lambda}\right)<\widehat{m}_{\lambda} \leq \widehat{\sigma}_{\lambda}\left(\widehat{u}_{\lambda}\right) .\right.
$$

From (3.48) we see that $\widehat{u}_{\lambda} \in \mathcal{S}(\lambda) \subseteq \operatorname{int} C_{+}$(see (3.42)) and $u_{\lambda} \neq \widehat{u}_{\lambda}$.

Next we examine what happens in the critical case $\lambda=\lambda_{*}$. 
Proposition 3.10. If hypotheses $\mathrm{H}(\mathrm{a})$ and $\left(\mathrm{H}_{1}\right)$ hold, then $\lambda_{*} \in \mathcal{L}$ and so, $\mathcal{L}=\left[\lambda_{*},+\infty\right)$.

Proof. Let $\left\{\lambda_{n}\right\}_{n \in \mathbb{N}} \subseteq \mathcal{L}$ and assume $\lambda_{n} \downarrow \lambda_{*}$. We can find $u_{n} \in \mathcal{S}\left(\lambda_{n}\right) \subseteq$ $\operatorname{int} C_{+}$such that $\varphi_{\lambda_{n}}\left(u_{n}\right)<0$ for all $n \geq 1$. Then, from the proof of Proposition 3.8, we know that $\left\{u_{n}\right\}_{n \in \mathbb{N}} \subseteq W^{1, p}(\Omega)$ is bounded. So, we may assume that

$$
u_{n} \stackrel{\mathrm{w}}{\longrightarrow} u_{*} \quad \text { in } W^{1, p}(\Omega) \quad \text { and } \quad u_{n} \rightarrow u_{*} \quad \text { in } L^{r}(\Omega) \text { as } n \rightarrow \infty .
$$

We have

$$
A\left(u_{n}\right)+\lambda_{n} u_{n}^{p-1}=N_{f}\left(u_{n}\right) \text { for all } n \geq 1 .
$$

Acting on (3.50) with $u_{n}-u_{*} \in W^{1, p}(\Omega)$, passing to the limit as $n \rightarrow \infty$ and using (3.49) we obtain $\lim _{n \rightarrow \infty}\left\langle A\left(u_{n}\right), u_{n}-u_{*}\right\rangle=0$, hence

$$
u_{n} \rightarrow u_{*} \quad \text { in } W^{1, p}(\Omega) \text { as } n \rightarrow \infty
$$

(see Proposition 2.6). Also, from the proof of Proposition 3.8), we know that $\widetilde{u}_{0} \leq u_{n}$ for all $n \geq 1$ (here $\widetilde{u}_{0} \in \operatorname{int} C_{+}$denotes the unique positive solution of the auxiliary problem (3.33)). Then from (3.51) we have $\widetilde{u}_{0} \leq u_{*}$, hence $u_{*} \neq 0$. If in (3.50) we pass to the limit as $n \rightarrow \infty$ and use (3.51), then

$$
A\left(u_{*}\right)+\lambda_{n} u_{*}^{p-1}=N_{f}\left(u_{*}\right),
$$

hence $u_{*} \in \mathcal{S}\left(\lambda_{*}\right) \subseteq \operatorname{int} C_{+}$and so $\lambda_{*} \in \mathcal{L}$, hence $\mathcal{L}=\left[\lambda_{*},+\infty\right)$.

In fact, we can show that for every $\lambda \in \mathcal{L}=\left[\lambda_{*},+\infty\right)$ problem $\left(\mathrm{P}_{\lambda}\right)$ admits a smallest positive solution $\bar{u}_{\lambda} \in \mathcal{S}(\lambda) \subseteq \operatorname{int} C_{+}$. We will need this fact in the next section where we produce nodal solutions.

Proposition 3.11. If hypotheses $\mathrm{H}(\mathrm{a})$ and $\left(\mathrm{H}_{1}\right)$ (resp. $\left(\mathrm{H}_{2}\right)$ ) hold, and $\lambda \in$ $\mathcal{L}=\left[\lambda_{*},+\infty\right)$, then problem $\left(\mathrm{P}_{\lambda}\right)$ admits a smallest positive solution $\bar{u}_{\lambda} \in \operatorname{int} C_{+}$ and the map $\lambda \mapsto \bar{u}_{\lambda}$ is nonincreasing (resp. decreasing) and right continuous from $\mathcal{L}$ into $C^{1}(\bar{\Omega})$.

Proof. As in Aizicovici, Papageorgiou and Staicu [2]), exploiting the monotonicity of $A$ (see Proposition 2.6), we see that for every $\lambda \in \mathcal{L}, \mathcal{S}(\lambda)$ is downward directed, that is, if $u_{1}, u_{2} \in \mathcal{S}(\lambda)$, there exists $u \in \mathcal{S}(\lambda)$ such that $u \leq u_{1}, u \leq u_{2}$.

Since we are looking for the smallest positive solution, and since $\mathcal{S}(\lambda)$ is downward directed, without any loss of generality, we may assume that there exists $C_{19}>0$ such that

$$
\|u\|_{\infty} \leq C_{19} \quad \text { for all } u \in W^{1, p}(\Omega) .
$$

From Hu and Papageorgiou [13, p. 178], we know that we can find $\left\{u_{n}\right\}_{n \in \mathbb{N}} \subseteq$ $\mathcal{S}(\lambda)$ such that $\inf \mathcal{S}(\lambda)=\inf _{n \geq 1} u_{n}$. We have

$$
A\left(u_{n}\right)+\lambda u_{n}^{p-1}=N_{f}\left(u_{n}\right) \text { for all } n \geq 1 .
$$


Because of (3.52), $\left\{u_{n}\right\}_{n \in \mathbb{N}} \subseteq W^{1, p}(\Omega)$ is bounded. So, we may assume that

$$
u_{n} \stackrel{\mathrm{w}}{\longrightarrow} \bar{u}_{\lambda} \quad \text { in } W^{1, p}(\Omega) \quad \text { and } \quad u_{n} \rightarrow \bar{u}_{\lambda} \quad \text { in } L^{r}(\Omega) \text { as } n \rightarrow \infty .
$$

Acting on (3.53) with $u_{n}-\bar{u}_{\lambda} \in W^{1, p}(\Omega)$, passing to the limit as $n \rightarrow \infty$ and using (3.54), we obtain $\lim _{n \rightarrow \infty}\left\langle A\left(u_{n}\right), u_{n}-\bar{u}_{\lambda}\right\rangle=0$, hence

$$
u_{n} \rightarrow \bar{u}_{\lambda} \text { in } W^{1, p}(\Omega) \text { as } n \rightarrow \infty .
$$

So, if in (3.53) we pass to the limit as $n \rightarrow \infty$ and use (3.55), then

$$
A\left(\bar{u}_{\lambda}\right)+\lambda\left(\bar{u}_{\lambda}\right)^{p-1}=N_{f}\left(\bar{u}_{\lambda}\right) .
$$

Recall that $\widetilde{u} \leq u_{n}$ for all $n \geq 1$, where $\widetilde{u} \in \operatorname{int} C_{+}$is the unique positive solution of problem (3.33) with $\lambda_{1}=\lambda$. Then, because of (3.55), we have $\widetilde{u}_{0} \leq \bar{u}_{\lambda}$, hence

$$
\bar{u}_{\lambda} \in \mathcal{S}(\lambda) \subseteq \operatorname{int} C_{+} \quad \text { and } \quad \bar{u}_{\lambda}=\inf \mathcal{S}(\lambda) .
$$

Next, let $\eta>\lambda$ and let $\bar{u}_{\lambda} \in \mathcal{S}(\lambda) \subseteq \operatorname{int} C_{+}$be the minimal positive solution of $\left(\mathrm{P}_{\lambda}\right)$. If hypotheses $\left(\mathrm{H}_{1}\right)$ (resp. $\left(\mathrm{H}_{2}\right)$ ) hold, then from Corollary 3.5 (resp. Proposition 3.7) we know that we can find $u_{\eta} \in \mathcal{S}(\eta)$ such that

$\bar{u}_{\lambda} \geq u_{\eta} \quad\left(\right.$ resp. $\left.\bar{u}_{\lambda}-u_{\eta} \in \operatorname{int} C_{+}\right) \quad$ hence $\quad \bar{u}_{\lambda} \geq \bar{u}_{\eta} \quad\left(\right.$ resp. $\left.\bar{u}_{\lambda}-\bar{u}_{\eta} \in \operatorname{int} C_{+}\right)$.

This proves the desired monotonicity of the map $\lambda \mapsto \bar{u}_{\lambda}$.

Finally, let $\left\{\lambda_{n}\right\}_{n \in \mathbb{N}} \subseteq \mathcal{L}$ be such that $\lambda_{n} \downarrow \lambda$. We have

$$
A\left(\bar{u}_{\lambda_{n}}\right)+\lambda_{n} \bar{u}_{\lambda_{n}}^{p-1}=N_{f}\left(\bar{u}_{\lambda_{n}}\right) \text { for all } n \geq 1 .
$$

From the proof of Proposition 3.8, we know that

$$
\left\{u_{\lambda_{n}}\right\}_{n \in \mathbb{N}} \subseteq W^{1, p}(\Omega) \text { is bounded. }
$$

Using (3.57), (3.58) and Proposition 2.6, as before, we can show that for at least a subsequence, we have

$$
\bar{u}_{\lambda_{n}} \rightarrow \bar{u} \quad \text { in } W^{1, p}(\Omega) \quad \text { and } \quad \bar{u} \in \mathcal{S}(\lambda) \subseteq \operatorname{int} C_{+} .
$$

We claim that $\bar{u}=\bar{u}_{\lambda} \in \operatorname{int} C_{+}$. From (3.58), Hu and Papageorgiou [14] (see Proposition 5) and the regularity result of Lieberman [15, p. 320], we know that we can find $\alpha \in(0,1)$ and $C_{20}>0$ such that

$$
u_{n} \in C^{1, \alpha}(\bar{\Omega}) \quad \text { and } \quad\left\|u_{n}\right\|_{C^{1, \alpha}(\bar{\Omega})} \leq C_{20} \quad \text { for all } n \geq 1 .
$$

Exploiting the compact embedding of $C^{1, \alpha}(\bar{\Omega})$ into $C^{1}(\bar{\Omega})$ and using (3.59), we infer that

$$
\bar{u}_{\lambda_{n}} \rightarrow \bar{u} \text { in } C^{1}(\bar{\Omega}) .
$$

If $\bar{u}_{\lambda} \neq \bar{u}$, then we can find $z_{0} \in \Omega$ such that $\bar{u}_{\lambda}\left(z_{0}\right) \neq \bar{u}\left(z_{0}\right)$, hence

$$
\bar{u}_{\lambda}\left(z_{0}\right)<\bar{u}_{\lambda_{n}} \text { for all } n \text { large enough (see (3.60)). }
$$


But from the previous part of the proof, we have $\bar{u}_{\lambda_{n}} \leq \bar{u}_{\lambda}$ for all $n \geq 1$ which contradicts (3.61). So, indeed $\bar{u}=\bar{u}_{\lambda}$ and we have proved the right continuity of $\lambda \mapsto \bar{u}_{\lambda}$ from $\mathcal{L}$ into $C^{1}(\bar{\Omega})$.

Summarizing the situation for problem $\left(\mathrm{P}_{\lambda}\right)$, we can state the following bifurcation-type result.

Theorem 3.12. (a) If hypotheses $\mathrm{H}(\mathrm{a})$ and $\left(\mathrm{H}_{1}\right)$ hold, then there exists $\lambda_{*}>0$ such that:

(i) For every $\lambda \in\left(0, \lambda_{*}\right)$, problem $\left(\mathrm{P}_{\lambda}\right)$ has no positive solutions.

(ii) For all $\lambda \geq \lambda_{*}$, problem $\left(\mathrm{P}_{\lambda}\right)$ has at least one positive solution. Moreover, for every $\lambda \geq \lambda_{*}$, problem $\left(\mathrm{P}_{\lambda}\right)$ has a smallest positive solution $\bar{u}_{\lambda} \in$ $\operatorname{int} C_{+}$and the map $\lambda \mapsto \bar{u}_{\lambda}$ from $\mathcal{L}$ into $C^{1}(\bar{\Omega})$ is nonincreasing and right continuous.

(b) If hypotheses $\mathrm{H}(\mathrm{a})$ and $\left(\mathrm{H}_{2}\right)$ hold, then there exists $\lambda_{*}>0$ such that:

(i) For every $\lambda \in\left(0, \lambda_{*}\right)$, problem $\left(\mathrm{P}_{\lambda}\right)$ has no positive solutions.

(ii) For $\lambda=\lambda_{*}$, problem $\left(\mathrm{P}_{\lambda}\right)$ has at least one positive solution $u_{*} \in \operatorname{int} C_{+}$.

(iii) For every $\lambda>\lambda_{*}$, problem $\left(\mathrm{P}_{\lambda}\right)$ has at least two positive solutions $u_{\lambda}$, $\widehat{u}_{\lambda} \in \operatorname{int} C_{+}, u_{\lambda} \neq \widehat{u}_{\lambda}$. Moreover, for every $\lambda \geq \lambda_{*}$, problem $\left(\mathrm{P}_{\lambda}\right)$ has a smallest positive solution $\bar{u}_{\lambda} \in \operatorname{int} C_{+}$and the map $\lambda \mapsto \bar{u}_{\lambda}$ from $\mathcal{L}$ into $C^{1}(\bar{\Omega})$ is decreasing and right continuous.

\section{Nodal solutions}

In this section, by imposing bilateral conditions on the reaction $f(z, \cdot)$ we produce nodal solutions. So, the new hypotheses on the reaction $f$ are the following:

$\left(\mathrm{H}_{3}\right) f: \Omega \times \mathbb{R} \rightarrow \mathbb{R}$ is a Carathéodory function such that for almost all $z \in \Omega$ $f(z, 0)=0, f(z, x) x>0$ for all $x \neq 0$ and

(i) there exists $a \in L^{\infty}(\Omega)_{+}$such that $|f(z, x)| \leq a(z)\left(1+|x|^{r-1}\right)$ for almost all $z \in \Omega$, all $x \in \mathbb{R}$, with $p<r<p^{*}$;

(ii) if $F(z, x)=\int_{0}^{x} f(z, s) d s$ then

$$
\lim _{x \rightarrow \pm \infty} \frac{F(z, x)}{|x|^{p}}=+\infty \quad \text { uniformly for a.a. } z \in \Omega ;
$$

(iii) there exist $\mu \in\left(\max \{(r-p) N / p, 1\}, p^{*}\right)$ and $\beta_{0}>0$ such that

$$
\beta_{0} \leq \liminf _{x \rightarrow \pm \infty} \frac{f(z, x) x-p F(z, x)}{|x|^{\mu}} \quad \text { uniformly for a.a. } z \in \Omega \text {; }
$$

(iv) there exists $\widehat{\delta}_{0}$ such that $0<q F(z, x) \leq f(z, x) x$ for almost all $z \in \Omega$, all $|x| \leq \widehat{\delta}_{0}$, and essinf $F\left(\cdot, \pm \widehat{\delta}_{0}\right)>0$ with $q \in(1, p)$ as in hypothesis $\mathrm{H}(\mathrm{a})$ (v). 
Remarks 4.1. Hypothesis $\left(\mathrm{H}_{3}\right)$ (iv) is a dual AR-condition near zero. It implies the weak condition

$$
\widehat{C}_{0}|x|^{q} \leq F(z, x) \quad \text { for a.a. } z \in \Omega \text {, all }|x| \leq \widehat{\delta}_{0} \text {, and some } \widehat{C}_{0}>0
$$

$($ see $[19]))$. So, now we have a stronger condition near zero (see hypothesis $\left.\left(\mathrm{H}_{3}\right)(\mathrm{iv})\right)$. Since the conditions on $f(z, \cdot)$ are now bilateral, reasoning as in Section 3 , we can find $\widehat{\lambda}_{*}>0$ such that for all $\lambda \geq \widehat{\lambda}_{*}$ problem $\left(\mathrm{P}_{\lambda}\right)$ has a biggest negative solution $\bar{v}_{\lambda} \in-\operatorname{int} C_{+}$(in this case the set of negative solutions of $\left(\mathrm{P}_{\lambda}\right)$ is upward directed, that is, if $v_{1}, v_{2}$ are negative solutions of $\left(\mathrm{P}_{\lambda}\right)$, then there exists a negative solution $v$ of $\left(\mathrm{P}_{\lambda}\right)$ such that $\left.v_{1} \leq v, v_{2} \leq v\right)$.

In what follows, we set $\widetilde{\lambda}_{*}=\max \left\{\lambda_{*}, \widehat{\lambda}_{*}\right\}$ (see Theorem 3.12). We have the following

THEOREM 4.2. If hypotheses $\mathrm{H}(\mathrm{a})$ and $\left(\mathrm{H}_{3}\right)$ hold and $\lambda \geq \widetilde{\lambda}_{*}$, then problem $\left(\mathrm{P}_{\lambda}\right)$ admits a nodal solution $y_{\lambda} \in C^{1}(\bar{\Omega})$.

Proof. Let $\bar{u}_{\lambda} \in \operatorname{int} C_{+}$and $\bar{v}_{\lambda} \in-\operatorname{int} C_{+}$be the two extremal constant sign solutions of $\left(\mathrm{P}_{\lambda}\right)$. We introduce the following Carathéodory function:

$$
e(z, x)= \begin{cases}f\left(z, \bar{v}_{\lambda}(z)\right) & \text { if } x<\bar{v}_{\lambda}(z), \\ f(z, x) & \text { if } \bar{v}_{\lambda}(z) \leq x \leq \bar{u}_{\lambda}(z), \\ f\left(z, \bar{u}_{\lambda}(z)\right) & \text { if } \bar{u}_{\lambda}(z)<x .\end{cases}
$$

Let $e_{ \pm}(z, x)=e\left(z, \pm x^{ \pm}\right)$(the positive and negative truncations of $\left.e(z, \cdot)\right)$. We set

$$
E(z, x)=\int_{0}^{x} e(z, s) d s, \quad E_{ \pm}(z, x)=\int_{0}^{x} e_{ \pm}(z, s) d s,
$$

and introduce the $C^{1}$-functionals $\psi_{\lambda}, \psi_{\lambda}^{ \pm}: W^{1, p}(\Omega) \rightarrow \mathbb{R}$ defined by

$$
\begin{aligned}
\psi_{\lambda}(u)=\int_{\Omega} G(D u(z)) d z+\frac{\lambda}{p}\|u\|_{p}^{p}-\int_{\Omega} E(z, u(z)) d z & \text { for all } u \in W^{1, p}(\Omega), \\
\psi_{\lambda}^{ \pm}(u)=\int_{\Omega} G(D u(z)) d z+\frac{\lambda}{p}\|u\|_{p}^{p}-\int_{\Omega} E_{ \pm}(z, u(z)) d z & \text { for all } u \in W^{1, p}(\Omega) .
\end{aligned}
$$

As before (see the proof of Proposition 3.4), we can show that

$$
K_{\psi_{\lambda}} \subseteq\left[\bar{v}_{\lambda}, \bar{u}_{\lambda}\right], \quad K_{\psi_{\lambda}^{+}} \subseteq\left[0, \bar{u}_{\lambda}\right], \quad K_{\psi_{\lambda}^{-}} \subseteq\left[\bar{v}_{\lambda}, 0\right]
$$

The extremality of $\bar{u}_{\lambda}$ and $\bar{v}_{\lambda}$ implies

$$
K_{\psi_{\lambda}} \subseteq\left[\bar{v}_{\lambda}, \bar{u}_{\lambda}\right], \quad K_{\psi_{\lambda}^{+}}=\left\{0, \bar{u}_{\lambda}\right\}, \quad K_{\psi_{\lambda}^{-}}=\left\{0, \bar{v}_{\lambda}\right\}
$$

Claim. $\bar{u}_{\lambda} \in \operatorname{int} C_{+}$and $\bar{v}_{\lambda} \in-\operatorname{int} C_{+}$are both local minimizers of $\psi_{\lambda}$.

It is clear from (4.2) that $\psi_{\lambda}^{+}$is coercive. Also, it is sequentially weakly lower semicontinuous. So, we can find $\bar{u} \in W^{1, p}(\Omega)$ such that

$$
\psi_{\lambda}^{+}(\bar{u})=\inf \left\{\psi_{\lambda}^{+}(u): u \in W^{1, p}(\Omega)\right\} .
$$


By (4.1) and since $q<p$, we see that $\psi_{\lambda}^{+}(\bar{u})<0=\psi_{\lambda}^{+}(0)$, hence $\bar{u} \neq 0$. From (4.3) and (4.4), it follows that $\bar{u}=\bar{u}_{\lambda} \in \operatorname{int} C_{+}$. Note that $\left.\psi_{\lambda}\right|_{C_{+}}=\left.\psi_{\lambda}^{+}\right|_{C_{+}}$, hence $\bar{u}_{\lambda} \in \operatorname{int} C_{+}$is a local $C^{1}(\bar{\Omega})$-minimizer of $\psi_{\lambda}$, therefore $\bar{u}_{\lambda} \in \operatorname{int} C_{+}$ is a local $W^{1, p}(\Omega)$-minimizer of $\psi_{\lambda}$ (see Proposition 2.7). Similarly for $\bar{v}_{\lambda} \in$ $-\operatorname{int} C_{+}$, using this time the functional $\psi_{\lambda}^{-}$. This proves Claim.

Without any loss of generality, we may assume that $\psi_{\lambda}\left(\bar{v}_{\lambda}\right) \leq \psi_{\lambda}\left(\bar{u}_{\lambda}\right)$ (the reasoning is similar if the opposite inequality holds).

We assume that $K_{\psi_{\lambda}}$ is finite (otherwise we already have infinitely many distinct nodal solutions, see (4.2) and (4.3)). By virtue of Claim, we can find $\rho \in(0,1)$ small enough such that

$$
\psi_{\lambda}\left(\bar{v}_{\lambda}\right) \leq \psi_{\lambda}\left(\bar{u}_{\lambda}\right)<\inf \left\{\psi_{\lambda}(u):\left\|u-\bar{u}_{\lambda}\right\|=\rho\right\}=: \bar{m}_{\lambda},\left\|\bar{v}_{\lambda}-\bar{u}_{\lambda}\right\|>\rho
$$

(see Aizicovici, Papageorgiou and Staicu [1], proof of Proposition 29). Recall that $\psi_{\lambda}$ is coercive. Therefore

$$
\psi_{\lambda} \text { satisfies the } C \text {-condition. }
$$

Then (4.5) and (4.6) permit the use of Theorem 2.1 (the mountain pass theorem). So, we can find $y_{\lambda} \in W^{1, p}(\Omega)$ such that

$$
y_{\lambda} \in K_{\psi_{\lambda}} \subseteq\left[\bar{v}_{\lambda}, \bar{u}_{\lambda}\right] \quad(\text { see }(4.3)) \quad \text { and } \quad \bar{m}_{\lambda} \leq \psi_{\lambda}\left(y_{\lambda}\right) .
$$

From (4.5) and (4.7) we see that $y_{\lambda} \in\left[\bar{v}_{\lambda}, \bar{u}_{\lambda}\right], y_{\lambda} \notin\left\{\bar{v}_{\lambda}, \bar{u}_{\lambda}\right\}$. Hence, if we show that $y_{\lambda} \neq 0$, then $y_{\lambda}$ is a nodal solution of $\left(\mathrm{P}_{\lambda}\right)$ (recall that $\bar{u}_{\lambda}$ and $\bar{v}_{\lambda}$ are the extremal constant sign solutions of $\left.\left(\mathrm{P}_{\lambda}\right)\right)$, and the nonlinear regularity of Lieberman [15] will imply that $y_{\lambda} \in C^{1}(\bar{\Omega})$.

Since $y_{\lambda}$ is a critical point of mountain pass type for $\psi_{\lambda}$, we have

$$
C_{1}\left(\psi_{\lambda}, y_{\lambda}\right) \neq 0
$$

(see Motreanu, Motreanu and Papageorgiou [19]). On the other hand, hypothesis $\left(\mathrm{H}_{3}\right)(\mathrm{iv})$ and the work of Marano and Papageorgiou [17], imply

$$
C_{k}\left(\psi_{\lambda}, 0\right)=0 \text { for all } k \geq 0 .
$$

From (4.8) and (4.9) it follows that $y_{\lambda} \neq 0$ and so $y_{\lambda} \in C^{1}(\bar{\Omega})$ is a nodal solution of $\left(\mathrm{P}_{\lambda}\right)$.

REMARKs 4.3. Nodal solutions for superlinear Neumann problems driven by the $p$-Laplacian were obtained by Aizicovici, Papageorgiou and Staicu in [2] (using the AR-condition) and in [3] (without the AR-condition). Theorem 4.2 improves substantially Theorem 3.5 in [18].

\section{REFERENCES}

[1] S. Aizicovici, N. S. Papageorgiou and V. Staicu, Degree theory for operators of monotone type and nonlinear elliptic equations with inequality constraints, Mem. Amer. Math. Soc. 196 (915), 2008. 
[2] _ Existence of multiple solutions with precise sign information for superlinear Neumann problems, Ann. Mat. Pura Appl. 188 (2009), 679-719.

[3] _ Multiple solutions for super-linear p-Laplacian Neumann problems, Osaka J. Math. 49 (2012), 699-740.

[4] _ Nodal solutions for $(p, 2)$-equations, Trans. Amer. Math. Soc. (in print), DOI: http://dx.doi.org/10.1090/S0002-9947-2014-06324-1

[5] _ Nodal solutions for nonlinear nonhomogeneous Neumann equations, Topol. Merthods Nonlinear Anal. 43 (2014), 421-438.

[6] L. Cherfils AND Y. ILYASOv, On the stationary solutions of generalized reaction-diffusion equations with $p$ and $q$ Laplacian, Commun. Pure Appl. Anal. 4 (2005), 9-22.

[7] S. Cingolani And M. Degiovanni, Nontrivial solutions for p-Laplacian equations with right hand side having p-linear growth, Comm. Partial Differential Equations 30 (2005), 1191-1203.

[8] D.G. Costa And C.A. Magalhães, Existence results for perturbations of the p-Laplacian, Nonlinear Anal. 24 (1995), 409-418.

[9] L. Damascelli, Comparison theorems for some quasilinear degenerate elliptic operators and applications to symmetry and monotonicity results, Ann. Inst. H. Poincaré Anal. Non Linéaire 15 (1998), 493-516.

[10] M. Filippakis, A. Kristaly and N. S. PApageorgiou, Existence of five nonzero solutions with constant sign for a p-Laplacian equation, Discrete Contin. Dyn. Syst. 24 (2009), 405440 .

[11] L. Gasinski and N. S. Papageorgiou, Nonlinear Analysis, Chapman \&Hall/ CRC Press, Boca Raton, 2006.

[12] _ Bifurcation-type results for nonlinear parametric elliptic equations, Proc. Royal Soc. Edinburgh Sect. A 142 (2012), 515-623.

[13] S. Hu And N. S. PApageorgiou, Handbook of Multivalued Analysis, Vol. I: Theory, Kluwer Academic Publishers, Dordrecht, The Netherland, 1997.

[14] _ Nonlinear Neumann equations driven by a nonhomogeneous differential operator, Commun. Pure Appl. Anal. 9 (2010), 1801-1827.

[15] G. Lieberman, The natural generalization of the natural conditions of Ladyzhenskaya and Ural'tseva for elliptic equations, Comm. Partial Differential Equations 16 (1991), $311-361$.

[16] G. LI AND C. YANG, The existence of a nontrivial solution to a nonlinear elliptic boundary value problem of $p$-Laplacian type without the Ambrosetti-Rabinawitz condition, Nonlinear Anal. 72 (2010), 4602-4613.

[17] S. Marano And N. S. PAPAgeorgiou, On the Neumann problem with p-Laplacian and noncoercitive resonant nonlinearity, Pacific J. Math. 253 (2011), 103-123.

[18] D. Motreanu, V. Motreanu and N.S. Papageorgiou, Multiple constant sign and nodal solutions for nonlinear Neumann eigenvalue problems, Ann. Scuola Norm. Sup. Pisa Cl. Sci. 10 (5) (2011), 729-755.

[19] _ Topological and Variational Methods with Applications to Nonlinear Boundary Value Problems, Springer, New York, 2014.

[20] D. Motreanu And N. S. Papageorgiou, Existence and multiplicity of solutions for Neumann problems, J. Differential Equations 232 (2007), 1-35.

[21] _ Multiple solutions for nonlinear Neumann problems driven by a nonhomogeneous differential operator, Proc. Amer. Math. Soc. 72 (2010), 4602-4613. 
[22] D. Mugnai And N.S. PApageorgiou, Wang's multiplicity result for superlinear $(p, q)$ equations without the Ambrosetti-Rabinowitz condition, Trans. Amer. Math. Soc. 366 (2014), 4919-4937.

[23] N.S. Papageorgiou And V.D. Radulescu, Qualitative phenomena for some classes of quaselinear elliptic equations with multiple resonance, Appl. Math. Optim. 69 (2014), 393-430.

[24] N.S. Papageorgiou, E.M. Rocha and V. Staicu, A multiplicity theorem for hemivariational inequalities with a p-Laplacian-like differential operator, Nonlinear Anal. 69 (2008), 1150-1163.

[25] P. Pucci And J. Serrin, The Maximum Principle, Birkhäuser, Basel, 2007.

[26] M. Sun, Multiplicity of solutions for a class of quasilinear elliptic equations at resonance, J. Math. Anal. Appl. 386 (2012), 661-668.

[27] P. Winkert, $L^{\infty}$-estimates for nonlinear elliptic Neumann boundary value problems, Nonlinear Differential Equations Appl. (NoDEA) 17 (2010), 289-302.

Manuscript received December 22, 2014 accepted April 7, 2015

Sergiu Aizicovici

Department of Mathematics

Ohio University

Athens, OH 45701, USA

E-mail address: aizicovs@ohio.edu

Nikolaos S. Papageorgiou

Department of Mathematics

National Technical University

Zografou Campus

Athens 15780, GREECE

E-mail address: npapg@math.ntua.gr

VASile Staicu

CIDMA and Department of Mathematics

University of Aveiro

Campus Universitário de Santiago

3810-193 Aveiro, PORTUGAL

E-mail address: vasile@ua.pt 